\title{
Cranberry juice concentrate does not significantly decrease the incidence of acquired bacteriuria in female hip fracture patients receiving urine catheter: a double-blind randomized trial
}

\author{
This article was published in the following Dove Press journal: \\ Clinical Interventions in Aging \\ 13 January 2017 \\ Number of times this article has been viewed
}

\author{
Anna-Karin Gunnarsson' \\ Lena Gunningberg² \\ Sune Larsson' \\ Kenneth B Jonsson' \\ 'Institution of Surgical Sciences, \\ Uppsala University, Uppsala, Sweden; \\ ${ }^{2}$ Institution of Public Health and \\ Caring Sciences, Uppsala University, \\ Uppsala, Sweden
}

\begin{abstract}
Background: Urinary tract infection (UTI) is a common complication among patients with hip fractures. Receiving an indwelling urinary catheter is a risk factor for developing UTIs. Treatment of symptomatic UTIs with antibiotics is expensive and can result in the development of antimicrobial resistance. Cranberries are thought to prevent UTI. There is no previous research on this potential effect in patients with hip fracture who receive urinary catheters.
\end{abstract}

Aim: The aim of this study is to investigate whether intake of cranberry juice concentrate preoperatively decreases the incidence of postoperative UTIs in hip fracture patients that received a urinary catheter.

Design: This study employed a randomized, placebo-controlled double-blind trial.

Method: Female patients, aged 60 years and older, with hip fracture $(n=227)$ were randomized to receive cranberry or placebo capsules daily, from admission, until 5 days postoperatively. Urine cultures were obtained at admission, 5 and 14 days postoperatively. In addition, Euro Qual five Dimensions assessments were performed and patients were screened for UTI symptoms. Result: In the intention-to-treat analysis, there was no difference between the groups in the proportion of patients with hospital-acquired postoperative positive urine cultures at any time point. When limiting the analysis to patients that ingested at least $80 \%$ of the prescribed capsules, 13 of $33(39 \%)$ in the placebo group and 13 of $47(28 \%)$ in the cranberry group $(P=0.270)$ had a positive urine culture at 5 days postoperatively. However, this difference was not statistically significant $(P=0.270)$.

Conclusion: Cranberry concentrate does not seem to effectively prevent UTIs in female patients with hip fracture and indwelling urinary catheter.

Keywords: urinary tract infection, elderly, randomized controlled trial, hospital, preventive, urine culture

\section{Introduction}

Patients with hip fracture are usually elderly, fragile, and at high risk of sustaining an adverse event. Pressure ulcers, renal failure, delirium, and urinary tract infections (UTIs) are common. ${ }^{1-4}$ The reported incidence of UTIs among hospital patients with hip fracture is high. ${ }^{4-6}$ UTI increases the risk of developing acute delirium, the length of hospital stay is prolonged, patients have a greater risk for subsequent falls, and quality of life deteriorates. ${ }^{5,7-10}$ Treating a symptomatic UTI with antibiotics is expensive, ${ }^{11}$ confers side effects, ${ }^{12}$ and can result in development of antimicrobial resistance. ${ }^{8}$ The overall cost to society is significant. ${ }^{13,14}$ For these reasons, there is a need for preventive measures to avoid UTIs in hospitalized patients.
Correspondence: Anna-Karin Gunnarsson

Institution of Surgical Sciences, Uppsala University Hospital, SE-75I 85 Uppsala, Sweden

$\mathrm{Tel}+46$ I8 6I| 448I

$\mathrm{Fax}+4618509427$

Email anna-karin.gunnarsson@ akademiska.se
Clinical Interventions in Aging 2017:12 137-143

(c) (1) (8) $\odot 2017$ Gunnarsson et al. This work is published and licensed by Dove Medical Press Limited. The full terms of this license are avalable at https://www.dovepress.com/terms.php cc. hereby accept the Terms. Non-commercial uses of the work are permitted without any further permission from Dove Medical Press Limited, provided the work is properly attributed. For peminsion for commercial use of this work, please see paragraphs 4.2 and 5 of our Terms (https://www.dovepress. com/terms.php). 
Cranberry derivatives are the most commonly used naturopathic drugs to prevent UTI. ${ }^{15-17}$ The mechanism of action may involve cranberry proanthocyanidin (PAC), which can prevent bacteria from attaching to the urinary epithelial cells. ${ }^{17-24}$ Previous studies indicated that cranberries reduce the occurrence of UTI in sexually active women with recurrent UTIs by $40 \%-50 \% .{ }^{25,26}$ However, in a study of hospitalized elderly men and women, no differences between the cranberry and placebo groups were found. ${ }^{27}$ A Cochrane review, ${ }^{1}$ which included seven trials, concluded that welldesigned, randomized, placebo-controlled trials are needed.

The aim of this study was to investigate whether cranberry capsules pre- and postoperatively decreased the incidence of bacteriuria among women with hip fracture receiving an indwelling urinary catheter.

\section{Methods}

\section{Design and study settings}

A randomized, placebo-controlled, double-blind, and parallel group study was conducted at Uppsala University Hospital in Sweden. The study took place between July 2009 and June 2013. The trial ended when the expiration date of the treatment capsules was reached.

\section{Participants}

Eligible participants were female patients over the age of 60 years with hip fracture. Exclusion criteria were: presence of a permanent indwelling urinary catheter, warfarin treatment, severe kidney or liver disease, high alcohol consumption, antibiotic treatment at arrival, or a general state of health making it unethical or unpractical to ask for participation. All patients received a preoperative indwelling urinary catheter and antibiotic prophylaxis to prevent wound infection. Planned removal of the urinary catheter was 2 days postoperatively; however, if the patient's medical condition required continued use of the catheter, it was removed later.

\section{Intervention}

The patients received two capsules of the study drug three times a day (t.i.d.) until 5 days postoperatively. Each capsule contained $550 \mathrm{mg}$ of cranberry powder (NurtiCran ${ }^{\circledR} 90$, Petefa AB, Västra Frölunda, Sweden) or placebo powder. The cranberry capsule contained $4.19 \mathrm{mg}$ of the putative active ingredient PAC. The first dose was given at least 30 minutes before the patient received the urinary catheter.

\section{Endpoint}

The primary endpoint in the study was a positive urinary culture at day 5 or 14 postoperatively among patients with sterile urine at admission. A positive urinary culture was defined as growth of a single urine pathogen of $>10^{4} \mathrm{cfu} / \mathrm{mL}$ urine specimen. ${ }^{28}$

The secondary endpoints were clinical symptoms of UTIs and health-related quality of life as measured by Euro Qual five Dimensions.

\section{Data collection}

The first sample was obtained at the time of catheterization. Subsequent urine samples were collected either as a midstream specimen or through the catheter, if still present.

Assessments of UTI symptoms and the Euro Qual five Dimensions were performed at inclusion, 5 and 14 days after surgery. To collect data on UTI symptoms, the patients were asked three questions: "Do you have a temperature?", "Do you have to urinate often?", and "Do you have pain on passing urine?".

\section{Randomization}

The patients were randomly assigned to one of two treatment groups by receiving a consecutive study number. The numbers correlated to prepared containers with study drugs that were independently and externally randomized in blocks of six by statistical programming. Cranberry and placebo capsules and containers were identical in appearance, blinded for both patients and health care professionals.

\section{Sample size and statistics}

A recent Swedish study reported that $52 \%$ of hip fracture patients contracted UTI during their hospital stay ${ }^{29}$ and that $12 \%$ of patients had UTI at admittance. A calculation to estimate the necessary sample size was based on these data. With $80 \%$ POWER to detect a decrease from $50 \%$ to $30 \%$ of hospital-acquired bacteriuria, using a significance level of $P<0.05$ and a two-sided test, 100 patients in each treatment group are required if $12 \%$ of patients have bacteriuria at admittance. A dropout rate of $20 \%$ was anticipated, which resulted in a goal of 125 patients in each treatment group.

To compare the proportions of bacteriuria and other dichotomous data between groups, a chi-square test was used. For continuous variables, a Student's $t$-test was used.

\section{Ethical considerations}

The regional Ethics Review Board in Uppsala (No 2007/287) and the Medical Products Agency (2008-002390) approved the study. Each patient signed an informed consent at the time of study inclusion. 


\section{Results}

\section{The patients and baseline data}

A total of 227 female patients with hip fracture were randomized. Nineteen patients were not followed because of unsuccessful initial urine sampling. Of the remaining 208 patients, 144 patients had a sterile urine sample at admittance and represent the intention-to-treat population in this study.

Included in the per-protocol analysis were patients who had taken $\geq 80 \%$ of the capsules. The per-protocol population included 92 patients, 40 in the placebo group and 52 in the cranberry group. In a subgroup analysis, we excluded patients that had received other antibiotics than prophylaxis. This group included 65 patients; 29 in the placebo group and 36 in the cranberry group (study flow chart and base-line patient characteristics in Figure S1 and Table S1).

The overall proportion of patients with a positive urine culture at the different sampling time points is shown in Figure 1A.

\section{The effect of cranberry Intention to treat}

Fifteen of 44 (34\%) patients in the placebo group and 14 of $53(26 \%)$ patients in the cranberry group had a positive urine culture at postoperative day 5. At day 14, 10 of 43 (23\%) patients in the placebo group and 12 of $49(24 \%)$ in the cranberry group had a positive urine culture. The proportion of patients with positive culture at either day 5 or day 14 postoperatively where 19 of $50(38 \%)$ and 23 of $61(38 \%)$, respectively $(P=0.975$, Relative Risk [RR] 0.988, 95\% CI 0.457-2.135) (Figure 1B).
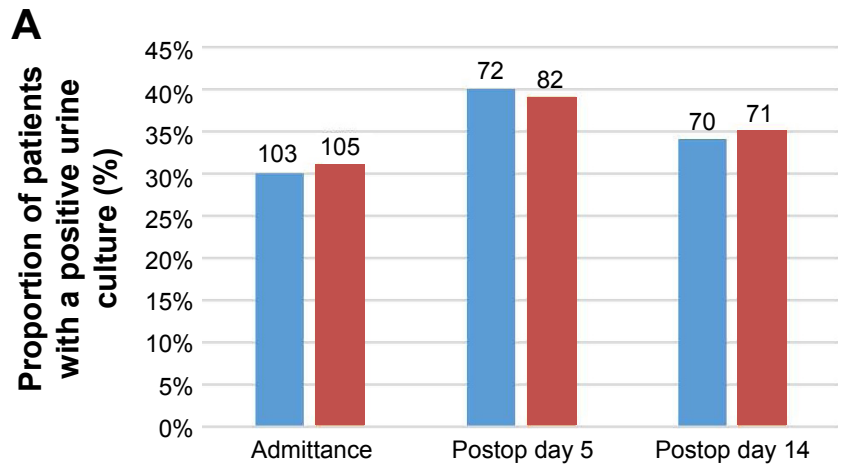

Placebo Cranberry

\section{Per protocol}

The number of patients who had a hospital-acquired positive urine culture 5 days postoperatively was 13 of 33 (39\%) in the placebo group and 13 of 47 (28\%) in the cranberry group $(P=0.270$; risk ratio [RR] $0.588 ; 95 \%$ confidence interval [CI] 0.288-1.516). Fourteen days postoperatively, the corresponding results were 9 of 33 (27\%) in the placebo group and 10 of $40(25 \%)$ in the cranberry group ( $P=0.826$; RR $0.889 ; 95 \%$ CI $0.312-2.536)$. The proportion of patients with a positive culture at either day 5 or day 14 postoperatively was 16 of $37(43 \%)$ in the placebo group and 20 of $52(38 \%)$ in the cranberry group $(P=0.651$, RR $0.820,95 \%$ CI $0.348-1.933)$ (Figure 2A).

\section{Per protocol and no antibiotics}

As antibiotic treatment strongly influences the results of urinary cultures, we also analyzed the data excluding all patients who were treated with other antibiotics than the perioperative prophylactics. The proportions of patients with positive urine cultures at the different time points are depicted in Figure 2B. The differences were not statistically significant.

\section{Discussion}

In this study, being the first to investigate the effect of cranberries in elderly women with hip fracture and urinary catheterization, we found no significant preventative effect of cranberry juice concentrate on hospital-acquired UTIs.

There are several explanations for these results and the discrepancies found in the literature. It is possible that, in spite of their long and frequent use for preventing UTIs,

B

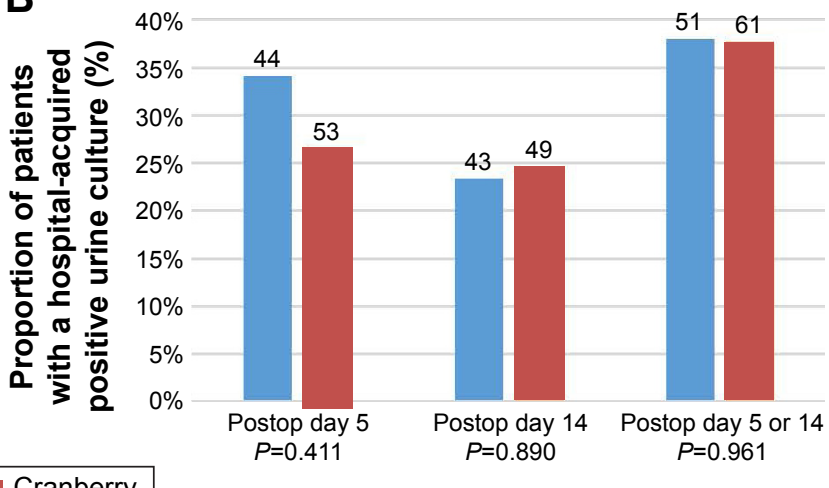

Figure I Proportion of patients with positive urine cultures.

Notes: (A) Percentage of all randomized patients with positive urine culture at admittance, five and 14 days postoperative. (B) Percentage of hospital-acquired positive urine cultures in the ITT population at 5 and 14 days postoperatively and at either 5 or 14 days postoperatively. The blue bars represent the placebo-treated patients and the red bars the cranberry-treated patients. The total number of patients in each group is shown above each bar. No significant difference between the groups was found using chi-square test.

Abbreviation: ITT, intention-to-treat. 
A

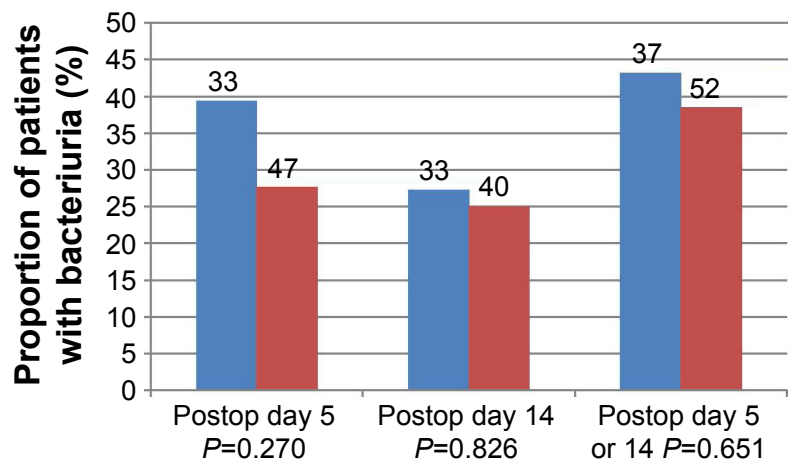

B

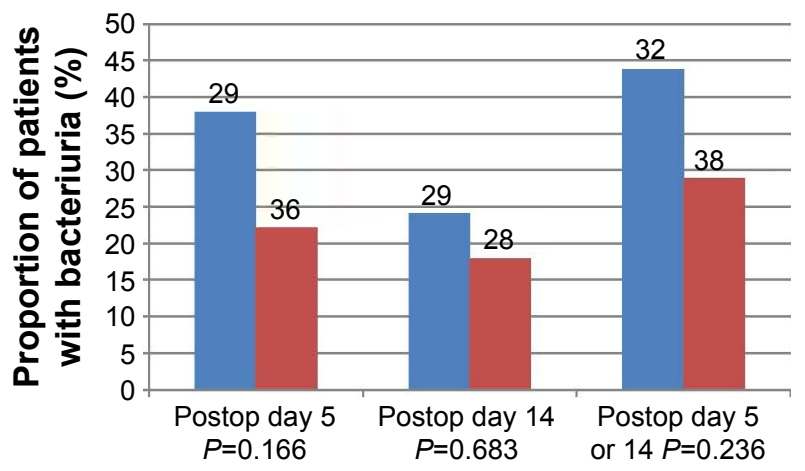

Placebo C Cranberry

Figure 2 Analysis of patients that had taken $>80 \%$ of administered capsules.

Notes: Percentage of hospital-acquired positive urine cultures at 5 and 14 days postoperatively and at either 5 or 14 days postoperatively. (A) shows the analysis in the total PP population and (B) shows the result when excluding patients who had taken antibiotics other than the prophylaxis during the study. The blue bars represent the placebotreated patients and the red bars the cranberry-treated patients. The total number of patients in each group is shown above each bar. No significant difference between the groups was found using chi-square test.

Abbreviation: PP, per-protocol.

cranberries lack a clinical effect for this indication and that other factors, such as an increase in fluid intake, explain previous findings. It is also possible that the pathogenesis of infections that are established during catheter use is different from those that occur in women with recurrent UTIs. A third explanation could be related to the timing and dose of the capsules in this study. In this study, patients received cranberry concentrate for 5 days. In the studies of recurrent UTIs in women, long-term treatments were used. In this study, the total daily intake was $25.2 \mu \mathrm{g}$ of PAC, which is equivalent to $200 \mathrm{~mL}$ of fresh cranberry juice and comparable to the amounts used in other studies. ${ }^{30}$ However, the amount of PAC given in this study was lower than the ideal dose found to prevent UTIs in sexually active women. ${ }^{30}$

We calculated that a sample size of 100 patients in each group would represent enough power to detect a 20-percentage point decrease in hospital-acquired UTIs. As the study progressed, however, a number of patients did not adhere to the protocol. Compared with the previous study, we also had a higher incidence of bacteriuria at admittance and a lower incidence of hospital-acquired UTIs, factors that unfortunately lowered the statistical power of the study.

\section{Conclusion}

In conclusion, this randomized-controlled study could not demonstrate a protective effect of cranberry concentrate on hospital-acquired UTIs in elderly women with hip fracture and urinary catheterization.

\section{Acknowledgments}

This study was funded by Uppsala University and Uppsala University Hospital, drugs were courtesy of Petefa AB, Sweden. This research was initially presented as part of AKG's doctoral thesis.

\section{Disclosure}

The authors report no conflicts of interest in this work.

\section{References}

1. Lindholm C, Sterner E, Romanelli M, et al. Hip fracture and pressure ulcers - the Pan-European Pressure Ulcer Study - intrinsic and extrinsic risk factors. Int Wound J. 2008;5(2):315-328.

2. Ulucay C, Eren Z, Kaspar EC, et al. Risk factors for acute kidney injury after hip fracture surgery in the elderly individuals. Geriatr Orthop Surg Rehabil. 2012;3(4):150-156.

3. Lundstrom M, Olofsson B, Stenvall M, et al. Postoperative delirium in old patients with femoral neck fracture: a randomized intervention study. Aging Clin Exp Res. 2007;19(3):178-186.

4. Poh KS, Lingaraj K. Complications and their risk factors following hip fracture surgery. J Orthop Surg (Hong Kong). 2013;21(2):154-157.

5. Kamel HK. The frequency and factors linked to a urinary tract infection coding in patients undergoing hip fracture surgery. J Am Med Dir Assoc. 2005;6(5):316-320.

6. Johansson I, Athlin E, Frykholm L, Bolinder H, Larsson G. Intermittent versus indwelling catheters for older patients with hip fractures. J Clin Nurs. 2002;11(5):651-656.

7. Eriksson I, Gustafson Y, Fagerstrom L, Olofsson B. Do urinary tract infections affect morale among very old women? Health Qual Life Outcomes. 2010;8:73.

8. Foxman B. Epidemiology of urinary tract infections: incidence, morbidity, and economic costs. Am J Med. 2002;113(Suppl 1A):5S-13S.

9. Eriksson I, Olofsson B, Gustafson Y, Fagerstrom L. Older women's experiences of suffering from urinary tract infections. J Clin Nurs. 2014; 23(9-10):1385-1394.

10. Sorbye LW, GrueEV. Hip fracture and urinary incontinence-use of indwelling catheter postsurgery. Scand J Caring Sci. 2013;27(3):632-642. 
11. Foxman B. Epidemiology of urinary tract infections: incidence, morbidity, and economic costs. Dis Mon. 2003;49(2):53-70.

12. Foxman B, Buxton M. Alternative approaches to conventional treatment of acute uncomplicated urinary tract infection in women. Curr Infect Dis Rep. 2013;15(2):124-129.

13. Foxman B, Barlow R, D'Arcy H, Gillespie B, Sobel JD. Urinary tract infection: self-reported incidence and associated costs. Ann Epidemiol. 2000;10(8):509-515.

14. Saint S. Clinical and economic consequences of nosocomial catheterrelated bacteriuria. Am J Infect Control. 2000;28(1):68-75.

15. The Cranberry Institute. Available from: http://www.cranberryinstitute. org. Accessed June 25, 2014.

16. Guay DR. Cranberry and urinary tract infections. Drugs. 2009;69(7): 775-807.

17. Krieger JN. Urinary tract infections: what's new? J Urol. 2002;168(6): 2351-2358.

18. Gupta K, Chou MY, Howell A, Wobbe C, Grady R, Stapleton AE. Cranberry products inhibit adherence of p-fimbriated Escherichia coli to primary cultured bladder and vaginal epithelial cells. J Urol. 2007; 177(6):2357-2360.

19. Jepson RG, Mihaljevic L, Craig J. Cranberries for preventing urinary tract infections. Cochrane Database Syst Rev. 2004(2):CD001321.

20. Di Martino P, Agniel R, David K, et al. Reduction of Escherichia coli adherence to uroepithelial bladder cells after consumption of cranberry juice: a double-blind randomized placebo-controlled cross-over trial. World J Urol. 2006;24(1):21-27.

21. Zafriri D, Ofek I, Adar R, Pocino M, Sharon N. Inhibitory activity of cranberry juice on adherence of type 1 and type P fimbriated Escherichia coli to eucaryotic cells. Antimicrob Agents Chemother. 1989; 33(1):92-98.

22. Schmidt DR, Sobota AE. An examination of the anti-adherence activity of cranberry juice on urinary and nonurinary bacterial isolates. Microbios. 1988;55(224-225):173-181.
23. Howell AB, Vorsa N, Der Marderosian A, Foo LY. Inhibition of the adherence of P-fimbriated Escherichia coli to uroepithelial-cell surfaces by proanthocyanidin extracts from cranberries. $N$ Engl J Med. 1998; 339(15):1085-1086.

24. Howell AB. Bioactive compounds in cranberries and their role in prevention of urinary tract infections. Mol Nutr Food Res. 2007;51(6): $732-737$.

25. Kontiokari T, Sundqvist K, Nuutinen M, Pokka T, Koskela M, Uhari M. Randomised trial of cranberry-lingonberry juice and Lactobacillus GG drink for the prevention of urinary tract infections in women. $B M J$. 2001;322(7302):1571.

26. Stothers L. A randomized trial to evaluate effectiveness and cost effectiveness of naturopathic cranberry products as prophylaxis against urinary tract infection in women. Can J Urol. 2002;9(3):1558-1562.

27. McMurdo ME, Bissett LY, Price RJ, Phillips G, Crombie IK. Does ingestion of cranberry juice reduce symptomatic urinary tract infections in older people in hospital? A double-blind, placebo-controlled trial. Age Ageing. 2005;34(3):256-261.

28. Aspevall O, Hallander H. Referensmetodik för laboratoriediagnostik vid kliniskt bakteriologiska laboratorier. In: Smittskyddsinstitutet, ed Vol I. Stockholm; 2000:22-40.

29. Halleberg Nyman M, Johansson JE, Persson K, Gustafsson M. A prospective study of nosocomial urinary tract infection in hip fracture patients. J Clin Nurs. 2011;20(17-18):2531-2539.

30. Howell AB, Botto H, Combescure C, et al. Dosage effect on uropathogenic Escherichia coli anti-adhesion activity in urine following consumption of cranberry powder standardized for proanthocyanidin content: a multicentric randomized double blind study. BMC Infect Dis 2010;10:94. 


\section{Supplementary materials}

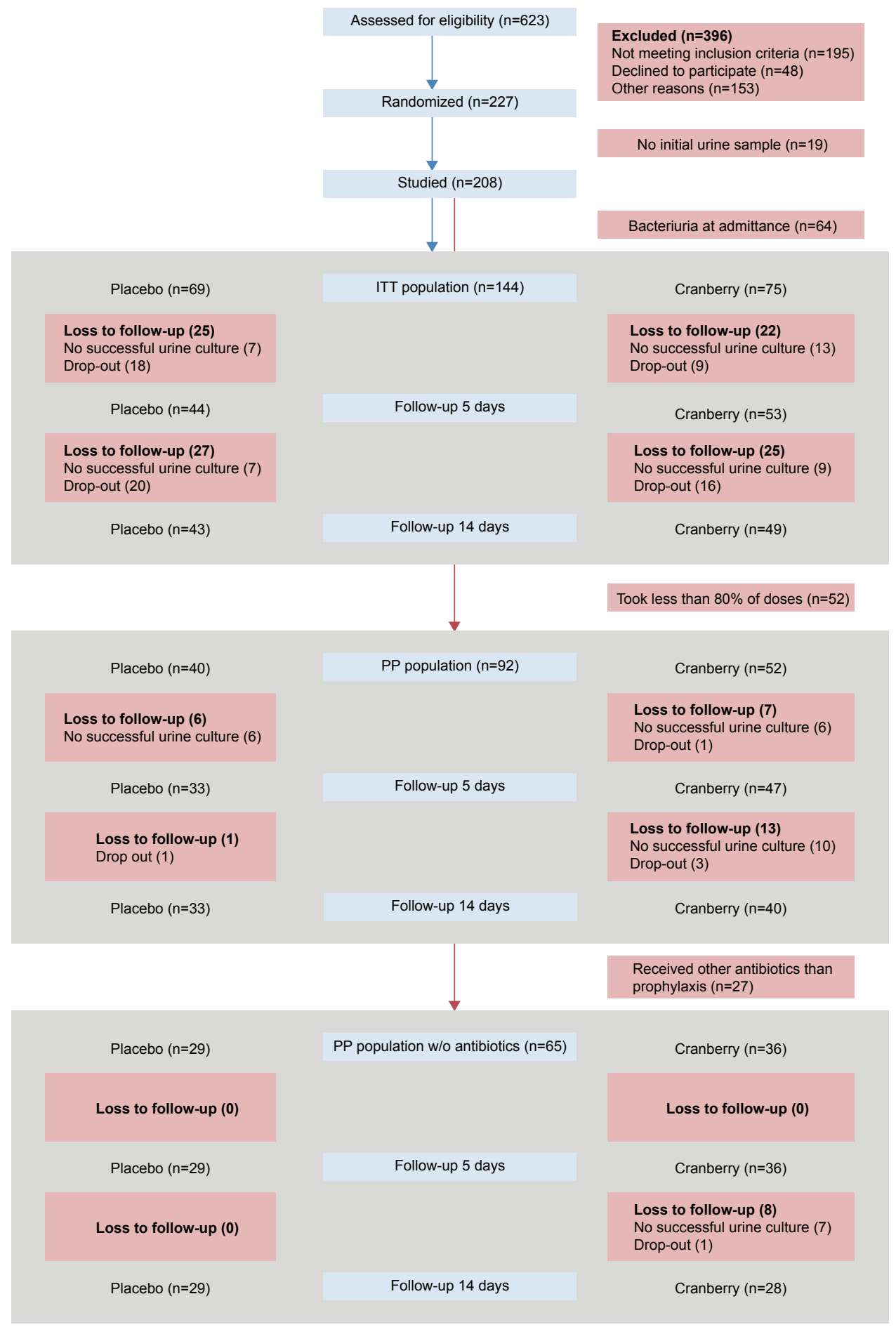

Figure SI Study flow chart.

Abbreviations: ITT, intention to treat; PP, per protocol. 
Table SI Baseline characteristics randomized patients

\begin{tabular}{|c|c|c|c|c|c|}
\hline Characteristics & Placebo group & $\mathbf{n}$ & Cranberry group & $\mathbf{n}$ & $P$-value \\
\hline Age mean (SD) & $82.6(8.9)$ & 114 & $83.1(8.6)$ & 113 & $0.667^{a}$ \\
\hline BMI mean (SD) & $24.4(4.8)$ & 93 & $23.1(4.0)$ & 102 & $0.038^{\mathrm{a}}$ \\
\hline SPMSQ mean(SD)[Md] & $7.59(3.0)[9]$ & 91 & 7.26 (3.4) Md 9 & 92 & $0.814^{b}$ \\
\hline \multicolumn{6}{|l|}{ UTI } \\
\hline UTI diagnosis last & 19 & 114 & 26 & 113 & $0.382^{c}$ \\
\hline \multicolumn{6}{|l|}{12 months } \\
\hline Positive culture at arrival & 31 & 103 & 33 & 105 & $0.835^{\mathrm{a}}$ \\
\hline \multicolumn{6}{|l|}{ Fracture type $^{d}$} \\
\hline Trochanteric & 52 & 114 & 60 & 113 & $0.739^{c}$ \\
\hline Cervical & 50 & 114 & 53 & 113 & \\
\hline \multicolumn{6}{|c|}{ Symptoms of ongoing UTI } \\
\hline High temperature & 4 & 114 & 6 & 113 & $0.586^{c}$ \\
\hline Urgency & 22 & 114 & 21 & 113 & $0.895^{c}$ \\
\hline Pain passing & 4 & 114 & 7 & 113 & $0.329^{c}$ \\
\hline
\end{tabular}

Notes: aStudent's t-test; 'Mann-Whitney U-test; 'chi-square test; ' 12 dropouts before surgery in the placebo group.

Abbreviations: SD, standard deviation; BMI, Body Mass Index; SPMSQ, Short Portable Mental Status Questionnaire; Md, median; UTI, Urinary Tract Infection.

\section{Publish your work in this journal}

Clinical Interventions in Aging is an international, peer-reviewed journal focusing on evidence-based reports on the value or lack thereof of treatments intended to prevent or delay the onset of maladaptive correlates of aging in human beings. This journal is indexed on PubMed Central, MedLine,

\section{Dovepress}

CAS, Scopus and the Elsevier Bibliographic databases. The manuscript management system is completely online and includes a very quick and fair peer-review system, which is all easy to use. Visit http://www.dovepress. $\mathrm{com} /$ testimonials.php to read real quotes from published authors. 